\title{
Reconsideration of Inferior Vena Cava Parameters for Estimating Right Atrial Pressure in an East Asian Population - Comparative Simultaneous Ultrasound-Catheterization Study -
}

\author{
Takayuki Kawata, MD; Masao Daimon, MD; Seitetsu L. Lee, MD; Koichi Kimura, MD; \\ Naoko Sawada, MD; Shuo-Ju Chiang, MD; Keitaro Mahara, MD; Takeshi Okubo, MD; \\ Tomoko Nakao, MD; Megumi Hirokawa, MD; Boqing Xu, MD; Tomoko S. Kato, MD; \\ Masafumi Watanabe, MD; Yutaka Yatomi, MD; Issei Komuro, MD
}

\begin{abstract}
Background: Ultrasound measurements of the inferior vena cava (IVC) diameter (IVCD), together with its respiratory variation, provide a noninvasive estimate of right atrial pressure (RAP). However, there is a paucity of studies that have compared this technique with simultaneous catheterization. We explored the best cut-off values of IVC parameters for elevated RAP in comparison with RAP measured by catheterization.

Methods and Results: We prospectively enrolled 120 East Asian patients who were scheduled for catheterization. The IVCD and IVC collapsibility index (IVCCI) were measured according to the current guidelines. The optimal maximum IVCD (IVCDmax) and IVCCI cut-offs for detecting elevated RAP (RAP $\geq 10 \mathrm{mmHg}$ ) were $17 \mathrm{~mm}$ and $40 \%$, respectively. When we combined both in proportion to the guidelines, the sensitivity and specificity for detecting elevated RAP were $75 \%$ and $94 \%$, respectively. When the cut-off values from the current guidelines (>21 mm and $<50 \%$ ) were applied, the respective sensitivity and specificity were $42 \%$ and $99 \%$. Interestingly, the cut-off value of the optimal IVCDmax indexed by body surface area $\left(11 \mathrm{~mm} / \mathrm{m}^{2}\right)$ was similar to previous Western population data. When we combined both cut-off values $\left(11 \mathrm{~mm} / \mathrm{m}^{2}\right.$ and $\left.40 \%\right)$, the sensitivity and specificity were $75 \%$ and $95 \%$, respectively.
\end{abstract}

Conclusions: The optimal absolute IVCDmax and IVCCI cut-offs to detect elevated RAP were smaller than those in the current guidelines. Indexed IVCDmax may be an IVC parameter that can be used internationally.

Key Words: Inferior vena cava diameter; Percent collapse; Right atrial pressure

A n elevated right atrial pressure (RAP) is a major prognostic predictor of morbidity and mortality in patients with pulmonary hypertension. ${ }^{1}$ Noninvasive assessment of RAP is used to estimate systolic pulmonary artery pressure in conjunction with the tricuspid regurgitation pressure gradient, and this assessment also plays a critical role in the management of volume control in patients with congestive heart failure. ${ }^{2}$

Currently, estimation of RAP using ultrasound measurements of the inferior vena cava (IVC) diameter (IVCD), together with its respiratory variation, is commonly performed, because it is simple and noninvasive and recommended in the current guidelines of the American Society of Echocardiography in conjunction with the European
Association of Echocardiography., ${ }^{3,4}$ However, in the clinical setting, sometimes the invasive RAP and RAP estimated from IVC parameters are not concordant. As a matter of fact, despite the widespread use of the IVC approach for RAP assessment, there are no previous studies that have evaluated this technique in comparison with simultaneous invasive methods..$^{5-8}$ There is no report that has compared invasive RAP with simultaneously obtained IVC parameters in a large cohort, and only a few small studies $^{9-11}$ have been published. The numbers of subjects in those studies were merely $35,{ }^{9} 71,{ }^{10}$ and $27 .{ }^{11}$ Moreover, most of the previous studies that support the current guidelines are derived from US and European populations. Although the current guidelines suggest a maximal IVCD

Received September 7, 2016; revised manuscript received November 23, 2016; accepted December 11, 2016; released online January 13, 2017 Time for primary review: 23 days

Department of Cardiovascular Medicine (T.K., S.L.L., K.K., N.S., M.H., M.W., I.K.), Department of Clinical Laboratory (M.D., T.N., B.X., Y.Y.), the University of Tokyo, Tokyo, Japan; Division of Cardiology, Department of Internal Medicine, School of Medicine, College of Medicine, Taipei Medical University, Taipei (S.-J.C.), Taiwan; Department of Cardiology, Sakakibara Heart Institute, Tokyo (K.M., T.O.); and Department of Cardiovascular Surgery, Juntendo University School of Medicine, Tokyo (T.S.K.), Japan

Mailing address: Takayuki Kawata, MD, Department of Cardiovascular Medicine, the University of Tokyo, 7-3-1 Hongo, Bunkyo-ku, Tokyo 113-8655, Japan. E-mail: dqh07724@nifty.ne.jp

ISSN-1346-9843 All rights are reserved to the Japanese Circulation Society. For permissions, please e-mail: cj@j-circ.or.jp 


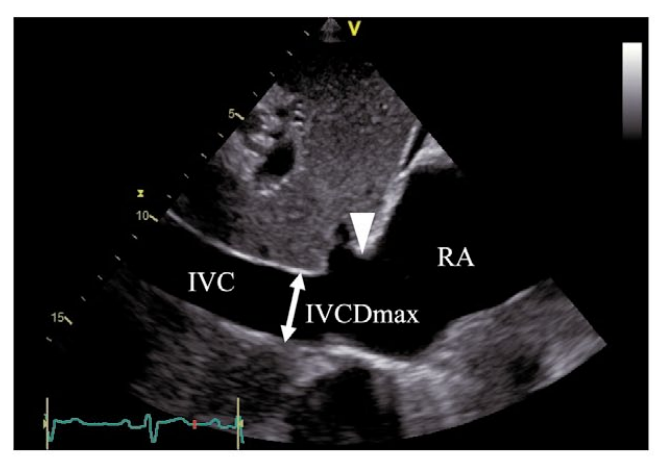

End-expiratory period

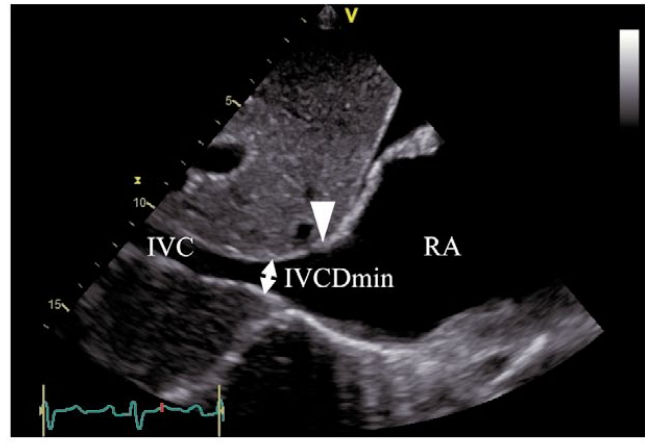

After brief sniff

Figure 1. Example of IVC ultrasound in the subcostal long axis view. Arrowhead indicates the IVC-RA junction. IVC, inferior vena cava; RA, right atrium.

$>21 \mathrm{~mm}$ in conjunction with an IVC collapse $<50 \%$ as cutoff thresholds for predicting RAP $\geq 10 \mathrm{mmHg}$, it is unclear whether these cut-off values in the current guidelines can be applied directly to Asian subjects. Recently, we reported that the cut-off values of IVC parameters to predict elevated RAP might be less than $21 \mathrm{~mm}$ and $50 \%$ in an Asian population. ${ }^{12}$ However, the major limitation of that study was that ultrasound and invasive measurement were not performed simultaneously. Although catheterization was performed within $24 \mathrm{~h}$ of IVC ultrasound, interpretation of the IVC parameters for RAP was limited. ${ }^{\mathbf{1 2}}$ Furthermore, a previous study, ${ }^{\mathbf{8}}$ as well as our study, ${ }^{\mathbf{1 2}}$ included patients with heart transplantation, and increased stiffness of the IVC at the join between the donor and recipient might have affected the IVC values or their respiratory variation. ${ }^{13}$

In the present study, we compared the invasive RAP value with IVC parameters that were obtained simultaneously and analyzed the optimal IVC parameter cut-off values to predict elevated RAP in a relatively large East Asian cohort.

\section{Methods}

\section{Study Subjects and Protocol}

The present study was a multicenter, cross-sectional study. Patients $(n=120)$ with heart disease who were scheduled for right-heart catheterization were prospectively enrolled from 3 institutions (University of Tokyo, Taipei Medical University and Sakakibara Heart Institute) between December 2014 and May 2016. Patients on mechanical ventilation and with heart transplantation were excluded. IVC ultrasound parameters were obtained during rightheart catheterization. The study protocol was approved by each institutional ethics committees, and written informed consent was given by all patients.

\section{Right-Heart Catheterization}

In all subjects, mean RAP was measured as RAP during the end-expiratory period using a flow-directed pulmonary artery catheter inserted via the femoral or internal jugular vein in the catheter laboratory. The observer was blinded to all echocardiographic data. All readings were referenced to the mid-axillary line with the patient in a supine posi-

\begin{tabular}{|lc|}
\hline \multicolumn{2}{|c|}{ Table 1. General Characteristics, Hemodynamic and } \\
Ultrasound Data of the Study Patients \\
Variable & $\mathbf{n}=120$ \\
Age, years & $54 \pm 17$ \\
Male (\%) & $60(50)$ \\
Height, cm & $161.2 \pm 9.4$ \\
Weight, kg & $57.8 \pm 12.9$ \\
BMI, kg/m ${ }^{2}$ & $22.1 \pm 3.8$ \\
BSA, m $^{2}$ & $1.6 \pm 0.2$ \\
Clinical diagnosis & \\
Pulmonary hypertension (\%) & $58(48)$ \\
Ischemic heart disease (\%) & $23(19)$ \\
Congenital heart disease (\%) & $19(16)$ \\
Cardiomyopathy (\%) & $9(8)$ \\
Valvular heart disease (\%) & $6(5)$ \\
Arrhythmia (\%) & $5(4)$ \\
Hemodynamic data & \\
SBP, mmHg & $118 \pm 20$ \\
DBP, mmHg & $63 \pm 13$ \\
HR, beats/min & $73 \pm 14$ \\
RAP, mmHg & $6 \pm 4$ \\
RAP $\geq 10 \mathrm{mmHg}(\%)$ & $12(10)$ \\
Ultrasound data & \\
IVCDmax, mm & $15 \pm 4$ \\
IVCDmin, mm & $8 \pm 4$ \\
IVCDmax/BSA, mm/m ${ }^{2}$ & $9 \pm 3$ \\
IVCDmin/BSA, mm/m² & $5 \pm 2$ \\
IVCCl, \% & $49 \pm 16$ \\
\hline
\end{tabular}

Data are presented as number (\%) or mean \pm SD. BMI, body mass index; BSA, body surface area; DBP, diastolic blood pressure; $\mathrm{HR}$, heart rate; IVC, inferior vena cava; IVCCI, IVC collapsibility index; IVCD, IVC diameter; RAP, mean right atrial pressure; $\mathrm{SBP}$, systolic blood pressure.

tion. Pressure calibration was performed before and after the pressure measurements. RAP measurements were performed during 5-10 cardiac cycles, and the mean value was calculated. 


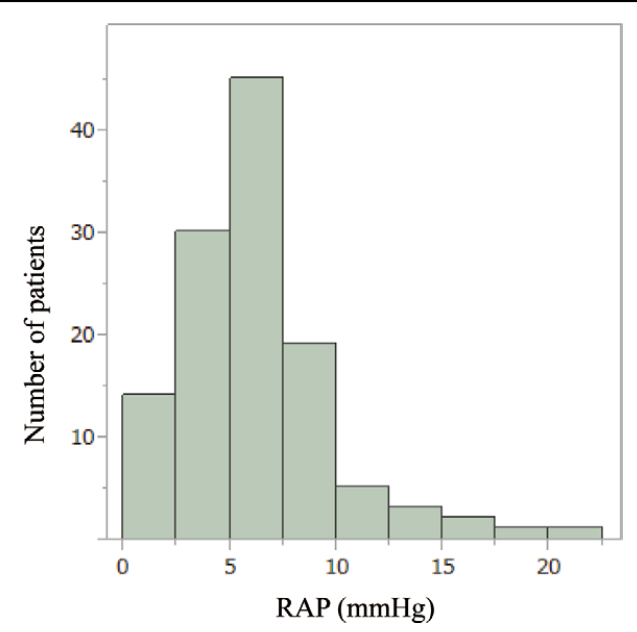

Figure 2. Distribution of right atrial pressure (RAP) obtained by right heart catheterization in the study population. The mean RAP is $6 \mathrm{mmHg}$.

\section{IVC Ultrasound}

IVC ultrasound was performed based on the current guidelines, ${ }^{3,4}$ with patients in the supine position in the catheter laboratory, by an experienced observer blinded to all invasive data. IVC images were recorded in a standard echocardiographic subcostal longitudinal view (Figure 1). The intrahepatic segment of the IVC was visualized as it entered the RA. All IVC dimensions were measured at the same sagittal level throughout the respiratory cycle. The maximum IVCD (IVCDmax) was measured $1.0-2.0 \mathrm{~cm}$ from the junction with the RA. ${ }^{3,4}$ Measurements were made perpendicular to the IVC long axis. Patients were then asked to sniff for the measurement of the minimum IVCD (IVCDmin). The IVC collapsibility index (IVCCI) with sniff respiration was computed using the following formula: [(IVCDmax-IVCDmin)/IVCDmax] $\times 100$. All values presented are the average of 3 consecutive measurements. Our echocardiography equipment and laboratory are maintained under the guidelines of the Japanese Society of Echocardiography. ${ }^{14}$

\section{Statistical Analysis}

All data are expressed as mean \pm standard deviation (SD) or the number of patients. A clinically significant elevation in RAP was defined as $\geq 10 \mathrm{mmHg}$. Receiver-operating characteristic (ROC) curves for each IVC parameter were generated to determine optimal cut-offs for the prediction of elevated RAP, as measured by catheterization. Using these cut-off values for the IVC parameters, we computed the sensitivity, specificity, positive predictive value and negative predictive value for elevated RAP. The cut-off values from the guidelines were also tested to calculate the accuracy of RAP estimation in our subjects. Comparison of the subjects' characteristics, IVC parameters and hemodynamic data between groups stratified according to BSA was performed with an unpaired t-test; a chi-square test was used for categorical variables. A probability value $<0.05$ was considered significant. All data were statistically analyzed using JMP version 11.0 (SAS Institute, Cary, NC, USA).

\section{Results}

\section{Patients' Characteristics}

Invasive data and IVC parameters were obtained for all patients $(n=120)$. Patients' general characteristics and clinical diagnosis are summarized in Table 1. The mean age of our cohort was 54 years, and half $(n=60)$ were male. The mean body mass index and BSA were $22.1 \mathrm{~kg} / \mathrm{m}^{2}$ and $1.6 \mathrm{~m}^{2}$, respectively. Indications for right-heart catheterization were as follows: pulmonary hypertension (48\%), ischemic heart disease $(19 \%)$, congenital heart disease $(16 \%)$, cardiomyopathy $(8 \%)$, valvular heart disease $(5 \%)$, and arrhythmia (4\%). Invasive and ultrasound data are also shown in Table 1. The mean RAP of the present cohort was $6 \mathrm{mmHg}$, and $12(10 \%)$ of 120 patients had RAP $\geq 10 \mathrm{mmHg}$. The mean IVCDmax, IVCDmin and IVCCI were $15 \mathrm{~mm}, 8 \mathrm{~mm}$ and $49 \%$, respectively. The distribution of invasive RAP in our study cohort is displayed in Figure 2.

\section{Estimation of RAP From IVC Parameters}

As with previous studies, all IVC parameters in the present study were weakly but significantly correlated with RAP (Table 2). According to the ROC curves, all the IVC parameters had an acceptable ability to detect elevated RAP (Table 2). The optimal IVCDmax cut-off value to detect RAP $\geq 10 \mathrm{mmHg}$ was $17 \mathrm{~mm}$ (sensitivity, $92 \%$; specificity, $76 \%$ ) and the optimal IVCCI cut-off was $40 \%$ (sensitivity, $75 \%$; specificity, $81 \%$ ). The representative ROC curves are shown in Figure 3. Both cut-off values were smaller than those recommended in the guidelines. ${ }^{3,4}$ The estimation of RAP $\geq 10 \mathrm{mmHg}$ on the basis of the IVCD and IVCCI in the proportions recommended in the guidelines are shown in Table 3. When both cut-off values $(>17 \mathrm{~mm}$ and $<40 \%$ ) were combined, the sensitivity and specificity for detecting RAP $\geq 10 \mathrm{mmHg}$ were $75 \%$ and $94 \%$, respectively, and this combination had an acceptable

\begin{tabular}{|c|c|c|c|c|c|c|c|c|}
\hline Variable & $\mathbf{r}$ & $P$ value & AUC & Cut-off & $\begin{array}{c}\text { Sensitivity } \\
(\%)\end{array}$ & $\begin{array}{c}\text { Specificity } \\
(\%)\end{array}$ & $\begin{array}{l}\text { PPV } \\
\text { (\%) }\end{array}$ & $\begin{array}{l}\text { NPV } \\
(\%)\end{array}$ \\
\hline IVCCI, \% & -0.40 & $<0.0001$ & 0.78 & 40 & 75 & 81 & 30 & 97 \\
\hline IVCDmax, mm & 0.47 & $<0.0001$ & 0.90 & 17 & 92 & 76 & 30 & 99 \\
\hline IVCDmin, mm & 0.55 & $<0.0001$ & 0.90 & 11 & 75 & 92 & 50 & 97 \\
\hline IVCDmax/BSA, $\mathrm{mm} / \mathrm{m}^{2}$ & 0.44 & $<0.0001$ & 0.89 & 11 & 92 & 78 & 31 & 99 \\
\hline IVCDmin/BSA, mm/m² & 0.54 & $<0.0001$ & 0.89 & 7 & 75 & 94 & 60 & 97 \\
\hline
\end{tabular}

AUC, area under the curve; PPV, positive predictive value; NPV, negative predictive value. Other abbreviations as in Table 1. 


\section{IVCDmax}

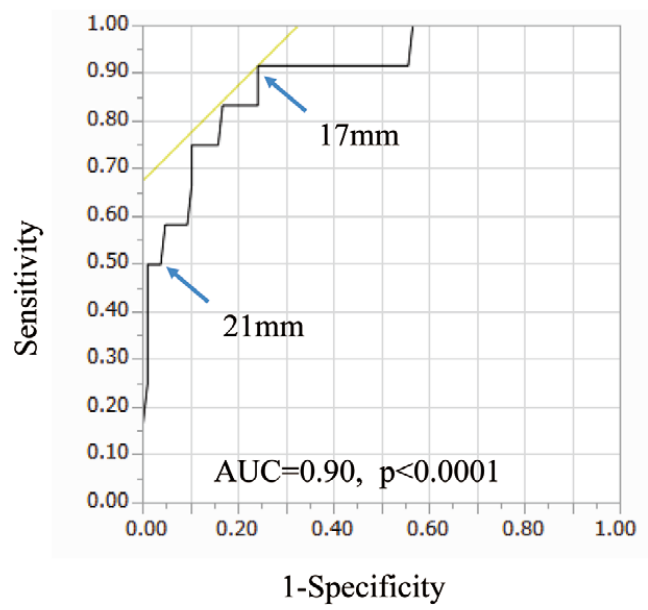

IVCCI

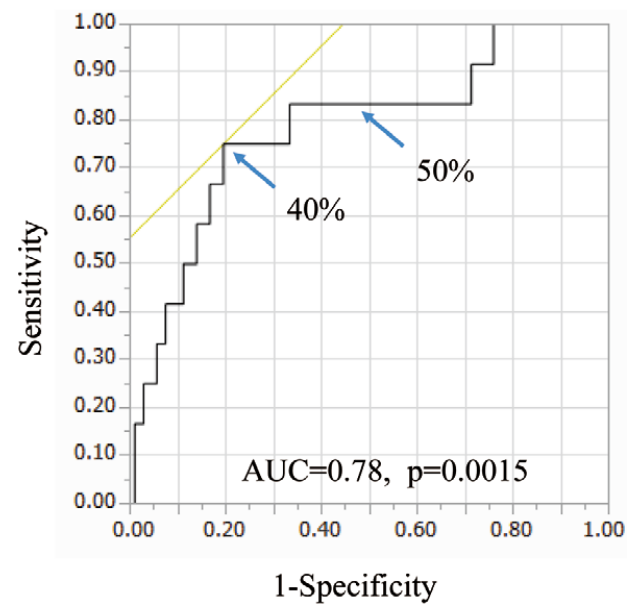

Figure 3. Receiver-operating characteristics curves of IVCDmax and IVCCI for predicting RAP $\geq 10 \mathrm{mmHg}$. Optimal cut-offs were $17 \mathrm{~mm}$ and $40 \%$, respectively, and those from the current guidelines are $21 \mathrm{~mm}$ and $50 \%$, respectively. IVC, inferior vena cava; IVCCI, IVC collapsibility index; IVCD, IVC diameter; RAP, right atrial pressure.

\begin{tabular}{|lcccc|}
\hline \multicolumn{2}{l}{ Table 3. Estimation of RAP $\geq 10$ mmHg on the Basis of IVCD and IVCCI } & & & \\
& $\begin{array}{c}\text { Sensitivity } \\
\text { (\%) }\end{array}$ & $\begin{array}{c}\text { Specificity } \\
\text { (\%) }\end{array}$ & $\begin{array}{c}\text { PPV } \\
\text { (\%) }\end{array}$ & $\begin{array}{c}\text { NPV } \\
\text { (\%) }\end{array}$ \\
IVCDmax $>17 m m$ and IVCCI $<40 \%$ & 75 & 94 & 56 & 97 \\
IVCDmax $/ \mathrm{BSA}>11 \mathrm{~mm} / \mathrm{m}^{2}$ and IVCCI $<40 \%$ & 75 & 95 & 64 & 97 \\
IVCDmax $>21 \mathrm{~mm}$ and IVCCI $<50 \%$ & 42 & 99 & 83 & 94 \\
\hline
\end{tabular}

Abbreviations as in Tables 1,2.

Table 4. Comparison of Larger $\left(>1.6 \mathrm{~m}^{2}\right)$ and Smaller BSA $\left(\leq 1.6 \mathrm{~m}^{2}\right)$ Subgroups

\begin{tabular}{|c|c|c|c|}
\hline Variable & $\begin{array}{l}\text { Larger BSA } \\
\quad(n=60)\end{array}$ & $\begin{array}{c}\text { Smaller BSA } \\
(n=60)\end{array}$ & $P$ value \\
\hline Age, years & $50 \pm 15$ & $59 \pm 19$ & 0.0088 \\
\hline Male (\%) & $45(75)$ & $15(33)$ & $<0.0001$ \\
\hline BMI, $\mathbf{k g} / \mathrm{m}^{2}$ & $23.8 \pm 4.0$ & $20.0 \pm 2.5$ & $<0.0001$ \\
\hline BSA, $\mathbf{m}^{2}$ & $1.8 \pm 0.1$ & $1.5 \pm 0.1$ & $<0.0001$ \\
\hline \multicolumn{4}{|l|}{ Hemodynamic data } \\
\hline $\mathrm{RAP}, \mathrm{mmHg}$ & $6 \pm 4$ & $6 \pm 3$ & 0.49 \\
\hline $\mathrm{RAP} \geq 10 \mathrm{mmHg}(\%)$ & $6(10)$ & $6(10)$ & 0.62 \\
\hline \multicolumn{4}{|l|}{ Ultrasound data } \\
\hline IVCDmax, mm & $16 \pm 4$ & $14 \pm 4$ & 0.012 \\
\hline IVCDmin, mm & $8 \pm 3$ & $7 \pm 4$ & 0.044 \\
\hline IVCDmax/BSA, mm/m² & $9 \pm 2$ & $9 \pm 3$ & 0.20 \\
\hline IVCDmin/BSA, mm/m² & $5 \pm 2$ & $5 \pm 3$ & 0.86 \\
\hline IVCCI, \% & $47 \pm 14$ & $51 \pm 16$ & 0.22 \\
\hline
\end{tabular}

Abbreviations as in Table 1.

ability to detect elevated RAP. On the other hand, when the cut-off values from the current guidelines $(>21 \mathrm{~mm}$ and $<50 \%$ ) were applied to our patients, the sensitivity and specificity for detecting RAP $\geq 10 \mathrm{mmHg}$ were $42 \%$ and $99 \%$, respectively, and the sensitivity was much lower than that obtained using our cut-off values (Table 3).

Interestingly, both the mean and cut-off values (for prediction of elevated RAP) of indexed IVCDmax (IVCDmax/BSA) and indexed IVCDmin (IVCDmin/BSA) were $9 \mathrm{~mm} / \mathrm{m}^{2}, 11 \mathrm{~mm} / \mathrm{m}^{2}, 5 \mathrm{~mm} / \mathrm{m}^{2}$ and $7 \mathrm{~mm} / \mathrm{m}^{2}$, all similar to previously published values in a Western population. ${ }^{8}$ When both IVCDmax/BSA $>11 \mathrm{~mm} / \mathrm{m}^{2}$ and IVCCI $<40 \%$ were combined, the sensitivity and specificity for detecting $\mathrm{RAP} \geq 10 \mathrm{mmHg}$ in our subjects were $75 \%$ and $95 \%$, respec- 


\begin{tabular}{|c|c|c|c|c|c|c|c|c|}
\hline \multirow[b]{2}{*}{ Variable } & \multicolumn{4}{|c|}{ Larger BSA } & \multicolumn{4}{|c|}{ Smaller BSA } \\
\hline & AUC & Cut-off & $\begin{array}{c}\text { Sensitivity } \\
(\%)\end{array}$ & $\begin{array}{c}\text { Specificity } \\
(\%)\end{array}$ & AUC & Cut-off & $\begin{array}{c}\text { Sensitivity } \\
(\%)\end{array}$ & $\begin{array}{c}\text { Specificity } \\
(\%)\end{array}$ \\
\hline IVCCI, \% & 0.70 & 44 & 80 & 59 & 0.84 & 38 & 86 & 87 \\
\hline IVCDmax, mm & 0.83 & 22 & 60 & 98 & 0.96 & 17 & 100 & 83 \\
\hline IVCDmin, mm & 0.87 & 10 & 80 & 80 & 0.92 & 11 & 86 & 91 \\
\hline IVCDmax/BSA, mm/m² & 0.84 & 11 & 80 & 91 & 0.94 & 12 & 86 & 93 \\
\hline IVCDmin/BSA, mm/m² & 0.85 & 8 & 60 & 98 & 0.92 & 7 & 86 & 89 \\
\hline
\end{tabular}

Abbreviations as in Tables 1,2.

tively (Table 3), and these values were adequate and similar to those obtained using IVCDmax $>17 \mathrm{~mm}$ and IVCCI $<40 \%$.

\section{Effect of BSA on IVC Parameters}

To explore the effect of BSA on IVC parameters, patients were divided into larger and smaller BSA subgroups according to the mean BSA $\left(1.6 \mathrm{~m}^{2}\right)$. Table 4 shows the comparison between subgroups with respect to patients' characteristics, hemodynamic and ultrasound data. As expected, there were more male and younger patients in the larger BSA subgroup. No significant difference in RAP was detected between the 2 groups. Both IVCDmax and IVCDmin were significantly greater in the larger BSA group, although no significant difference in IVCCI was found between groups. There was no significant difference with respect to IVCDmax/BSA between the 2 groups. Optimal cut-offs for detecting elevated RAP were also estimated for both the larger and the smaller BSA subgroups from respective ROC curves, and that of IVCDmax was $22 \mathrm{~mm}$ in the larger BSA group and $17 \mathrm{~mm}$ in the smaller BSA group (Table 5). However, the cut-off value of IVCDmax/BSA for predicting elevated RAP in both subgroups was almost similar (11 and $\left.12 \mathrm{~mm} / \mathrm{m}^{2}\right)$, and those of IVCCI in both subgroups were approximately 40\% (Table 5).

\section{Discussion}

The key finding of the present study was that the optimal absolute IVCDmax and IVCCI cut-offs to detect elevated RAP (RAP $\geq 10 \mathrm{mmHg}$ ) were $17 \mathrm{~mm}$ and $40 \%$, respectively, in this East Asian population, and both values were smaller than those in the current guidelines.

To the best of our knowledge, the present study is the largest to compare IVC ultrasound parameters simultaneously with invasive RAP. Although several previous studies generated data that support the current guidelines, $\mathbf{3 , 4}$ they had either small numbers of patients and/or the IVC parameters were not measured at the same time as invasive RAP; thus, the current guidelines may not be appropriate. We use IVC ultrasound parameters in daily practice according to the current guidelines. ${ }^{3,4}$ However, sometimes there is discrepancy between the RAP estimated from the IVC parameters and the RAP measured invasively in the clinical setting. This difference may lead to false hemodynamic assessment and false estimation of pulmonary artery pressures. Generally, the normal range for RAP is assumed to be between 1 and $7 \mathrm{mmHg} .{ }^{15}$ Because RAP is equivalent to a lowest intracardiac pressure, we should estimate it carefully because both RAP and IVC parameters may influenced largely by several factors and measurement conditions. Previous studies have demonstrated that intravenous fluid administration ${ }^{\mathbf{1 6}}$ or intravenous bolus injection of diuretic ${ }^{17}$ can change the IVCD significantly. Those results suggest that changes in intravenous fluid volume promptly affect IVCD or RAP, and the time lag between catheter and ultrasound study may result in the discrepancy between invasive measurement of RAP and that estimated from the IVC parameters. Moreover, the size of the IVC is affected by patient's position. ${ }^{6}$ Therefore, we obtained IVC parameters at the same time and in the same position as for the invasive RAP measurements to avoid these influential factors in a large number of patients. Nevertheless, both IVCD and IVCCI significantly, but only weakly, correlated with RAP as shown in Table 2. Previous reports that compared invasive RAP with simultaneously obtained IVC parameters also have only a moderate correlation coefficient $(\mathrm{r}=0.35-0.76) .{ }^{9-11}$ In light of these findings and our results, it might be difficult to estimate an absolute value of RAP from only the IVC parameters. Thus, IVCD and IVCCI can be used to detect elevated RAP, but we also need to recognize that they have limited utility in identifying the magnitude of the elevation, and a comprehensive estimate of RAP through consideration of physical findings and other echocardiographic parameters, such as tricuspid E/e', as in the current guidelines, ${ }^{3,4}$ is suggested. In this study, to detect elevated RAP, we used ROC curve analysis and found that the optimal absolute cut-off value of IVCDmax was $17 \mathrm{~mm}$, less than the recommended $21 \mathrm{~mm}$. From our ROC curve, a cut-off value of $21 \mathrm{~mm}$ had a sensitivity of $50 \%$ and specificity of $97 \%$ (Figure 3), and this sensitivity was very low, as we had previously published. ${ }^{\mathbf{1 2}}$ A new classification scheme may be required based on these results.

However, it must be recognized that our population was East Asian, because we encounter mainly East Asian patients in our daily practice. Our East Asian subjects had a smaller BSA compared with subjects in Western populations. Because organ size is thought to vary with body size (there are racial differences in body size), several echocardiographic variables are indexed to BSA. The absolute values of IVC parameters have been used worldwide without regard to differences in body size among races. Therefore, it is a possibility that the difference in body size is a reason for the discrepancy between IVCD and invasive RAP when we used the cut-off values from the current guidelines in our East Asian patients. Actually, the cut-off value of IVCDmax/BSA (for the prediction of high RAP) in our East Asian subjects was $11 \mathrm{~mm} / \mathrm{m}^{2}$, which is in 
accordance with previous values from a study in the USA. ${ }^{8,18}$ Moreover, the cut-off value of IVCDmax in the present larger BSA subgroup was $22 \mathrm{~mm}$, an almost similar value from the current Western guidelines. ${ }^{\mathbf{3}, \mathbf{4}}$ Recently, Taniguchi et al reported that body size may be an important consideration when IVCD is used for assessing RAP; their optimal cut-off point of IVCDmax was $21 \mathrm{~mm}$ for patients with larger BSA and $17 \mathrm{~mm}$ for those with smaller BSA. ${ }^{19}$ Our results also showed that IVCD was greater in patients with larger BSA than in the smaller BSA group, although no difference in RAP was found in either group. On the other hand, the cut-off value of IVCDmax/BSA for predicting elevated RAP in both groups was almost similar. Because both IVCDmax and IVCDmax/BSA had similar accuracy in predicting elevated RAP in this study and prior reports, ${ }^{\mathbf{8} 12}$ IVCDmax/BSA should probably be used worldwide instead of the absolute IVCDmax to negate any influence of body size.

Interestingly, there was no remarkable difference with respect to the IVCCI cut-off between our results (40\%) and several previous studies in Western populations. Brennan et al found that using ROC analysis, the optimal IVCCI cut-off by sniff was $40 \%$ to predict RAP $\geq 10 \mathrm{mmHg}{ }^{8}$ Moreno et al reported that an IVCCI of $40 \%$ can clearly separate patients with elevated RAP $(\geq 7 \mathrm{mmHg})$ from normal RAP. 5 Patel et al evaluated the utility of 3-dimensional RA volume index to predict elevated RAP, and they used IVCCI cut-off of $40 \%$ as a reliable IVC parameter. ${ }^{20}$ The IVC collapses during inspiration because of negative intrathoracic pressure related to inspiration. Taniguchi et al reported that the cut-off points determined for respirophasic changes were not affected by body size. ${ }^{19}$ There was also no significant difference in IVCCI between the larger and smaller BSA subgroups in the present study. These results, as well as our findings, suggest that the respiratory force during inspiration is not affected by body size; therefore, IVCCI should be similar among the various races. On the other hand, both the current guidelines ${ }^{3,4}$ and Kircher et al ${ }^{7}$ recommend an IVCCI of $50 \%$ for predicting elevated RAP. At least from our results, there was a large change in specificity (to detect elevated RAP) between an IVCCI of 40\% and 50\% (Figure 3), whereas sensitivity was similar. This may be the reason why we are apt to estimate a higher RAP with an IVCCI of $50 \%$ compared with the invasive method. Our results suggested that the appropriate cut-off value for IVCCI is $40 \%$, as already shown in several previous reports. This is important, considering our study has been the largest yet to compare IVCCI with invasive RAP measured simultaneously.

\section{Study Limitations}

There are several important limitations to the present study. First, the echocardiographic data acquisition in this study was limited to the IVC; thus, complete echocardiographic data were not acquired. This prevents assessment of confounding factors that might affect IVC size and respirophasic changes such as tricuspid regurgitation or assessment of the additive information from other echocardiographic parameters. In particular, tricuspid regurgitation is thought to be a major confounding factor, and we tried to evaluate the severity of tricuspid regurgitation. However, we could not obtain appropriate images because of limited evaluation time and patient's position, and we were concerned about erroneous grading of tricuspid regurgitation. Further investigations are needed to clarify the effect of significant tricuspid regurgitation on IVC parameters. Second, all subjects were referred for clinically indicated reasons, so our study population included only patients with cardiac disease. It is unclear whether our results can be applied to healthy subjects. In addition, unlike in previous reports, most of our patients had pulmonary hypertension or congenital heart disease. However, it is more important to obtain an accurate estimate of RAP in a population such as ours than in healthier subjects.

\section{Conclusion}

From this comparative, simultaneous, ultrasound-catheterization study in an East Asian population, the optimal absolute IVCDmax and IVCCI cut-off values to detect elevated RAP were $17 \mathrm{~mm}$ and $40 \%$, respectively, and these values are smaller than those in the current guidelines., Therefore, a new classification scheme may be needed to estimate RAP more accurately. Otherwise, IVCD indexed by BSA may provide an internationally applicable cut-off value for elevated RAP, regardless of race, by negating the influence of body size. A large prospective cohort study in a multiracial population is needed to validate our findings.

\section{Acknowledgments}

This work was partially supported by a Grant-in-Aid for Scientific Research C (15K01326) from the Japan Society for the Promotion of Science (Takayuki Kawata).

\section{References}

1. Sandoval J, Bauerle O, Palomar A, Gómez A, Martínez-Guerra ML, Beltrán M, et al. Survival in primary pulmonary hypertension: Validation of a prognostic equation. Circulation 1994; 89: $1733-1744$.

2. Pellicori P, Carubelli V, Zhang J, Castiello T, Sherwi N, Clark $\mathrm{AL}$, et al. IVC diameter in patients with chronic heart failure: Relationships and prognostic significance. JACC Cardiovasc Imaging 2013; 6: 16-28.

3. Rudski LG, Lai WW, Afilalo J, Hua L, Handschumacher MD, Chandrasekaran K, et al. Guidelines for the echocardiographic assessment of the right heart in adults: A report from the American Society of Echocardiography endorsed by the European Association of Echocardiography, a registered branch of the European Society of Cardiology, and the Canadian Society of Echocardiography. $J$ Am Soc Echocardiogr 2010; 23: 685-713.

4. Lang RM, Badano LP, Mor-Avi V, Afilalo J, Armstrong A, Ernande L, et al. Recommendations for cardiac chamber quantification by echocardiography in adults: An update from the American Society of Echocardiography and the European Association of Cardiovascular Imaging. $J$ Am Soc Echocardiogr 2015; 28: $1-39$.

5. Moreno FL, Hagan AD, Holmen JR, Pryor TA, Strickland RD, Castle $\mathrm{CH}$. Evaluation of size and dynamics of the inferior vena cava as an index of right-sided cardiac function. Am J Cardiol 1984; 53: 579-585.

6. Nakao S, Come PC, McKay RG, Ransil BJ. Effects of positional changes on inferior vena caval size and dynamics and correlations with right-sided cardiac pressure. Am J Cardiol 1987; 59: $125-132$.

7. Kircher BJ, Himelman RB, Schiller NB. Noninvasive estimation of right atrial pressure from the inspiratory collapse of the inferior vena cava. Am J Cardiol 1990; 66: 493-496.

8. Brennan JM, Blair JE, Goonewardena S, Ronan A, Shah D, Vasaiwala S, et al. Reappraisal of the use of inferior vena cava for estimating right atrial pressure. J Am Soc Echocardiogr 2007; 20: $857-861$.

9. Nagueh SF, Kopelen HA, Zoghbi WA. Relation of mean right atrial pressure to echocardiographic and Doppler parameters of right atrial and right ventricular function. Circulation 1996; 93: $1160-1169$

10. Ommen SR, Nishimura RA, Hurrell DG, Klarich KW. Assessment of right atrial pressure with 2-dimensional and Doppler echocardiography: A simultaneous catheterization and echocardiography study. Mayo Clin Proc 2000; 75: 24-29. 
11. Simonson JS, Schiller NB. Sonospirometry: A new method for noninvasive estimation of mean right atrial pressure based on two-dimensional echocardiographic measurements of the inferior vena cava during measured inspiration. $\mathrm{J} \mathrm{Am} \mathrm{Coll} \mathrm{Cardiol}$ 1988; 11: 557-564

12. Lee SL, Daimon M, Kawata T, Kohro T, Kimura K, Nakao T, et al. Estimation of right atrial pressure on inferior vena cava ultrasound in Asian patients. Circ J 2014; 78: 962-966.

13. Badano LP, Miglioranza MH, Edvardsen T, Colafranceschi AS, Muraru D, Bacal F, et al. European Association of Cardiovascular Imaging/Cardiovascular Imaging Department of the Brazilian Society of Cardiology recommendations for the use of cardiac imaging to assess and follow patients after heart transplantation. Eur Heart J Cardiovasc Imaging 2015; 16: 919-948.

14. Nakatani S, Akaishi M, Asanuma T, Hashimoto S, Izumi C, Iwanaga $\mathrm{S}$, et al. Guidelines from the Japanese Society of Echocardiography: Guidance for the management and maintenance of echocardiography equipment. $J$ Echocardiogr 2015; 13: $1-5$.

15. Davidson CJ, Bonow RO. Cardiac catheterization. In: Bonow RO, Mann DL, Zipes DP, Libby P, editors. Braunwald's heart disease: A textbook of cardiovascular medicine. 9th edn. New
York: Elsevier; 2012; 383-405.

16. Celebi Yamanoğlu NG, Yamanoğlu A, Parlak İ, Pınar P, Tosun A, Erkuran B, et al. The role of inferior vena cava diameter in volume status monitoring; the best sonographic measurement method? Am J Emerg Med 2015; 33: 433-438.

17. Tchernodrinski S, Lucas BP, Athavale A, Candotti C, Margeta B, Katz A, et al. Inferior vena cava diameter change after intravenous furosemide in patients diagnosed with acute decompensated heart failure. J Clin Ultrasound 2015; 43: 187-193.

18. Mintz GS, Kotler MN, Parry WR, Iskandrian AS, Kane SA. Real-time inferior vena caval ultrasonography: Normal and abnormal findings and its use in assessing right-heart function. Circulation 1981; 64: 1018-1025.

19. Taniguchi T, Ohtani T, Nakatani S, Hayashi K, Yamaguchi O, Komuro I, et al. Impact of body size on inferior vena cava parameters for estimating right atrial pressure: A need for standardization? J Am Soc Echocardiogr 2015; 28: 1420-1427.

20. Patel AR, Alsheikh-Ali AA, Mukherjee J, Evangelista A, Quraini $\mathrm{D}$, Ordway $\mathrm{LJ}$, et al. 3D echocardiography to evaluate right atrial pressure in acutely decompensated heart failure correlation with invasive hemodynamics. JACC Cardiovasc Imaging 2011; 4: 938-945. 